Running Title: Neurofilament Light and Mild Brain Injury

\title{
Serum Neurofilament Light is Elevated Differentially in Older Adults with Uncomplicated Mild Traumatic Brain Injuries
}

\author{
Grant L. Iverson, Ph.D. \\ Department of Physical Medicine and Rehabilitation, Harvard Medical School; \\ Spaulding Rehabilitation Hospital; \& Home Base, A Red Sox Foundation and Massachusetts \\ General Hospital Program, Boston, Massachusetts, USA. \\ Email: giverson@mgh.harvard.edu \\ Address: Center for Health and Rehabilitation Research, Department of Physical Medicine \& \\ Rehabilitation, Harvard Medical School, 79/96 Thirteenth Street, Charlestown Navy Yard, \\ Charlestown, MA, 02129, USA.
Preethi J. Reddi
Department of Biology, Emory University, Atlanta, GA, USA
Email: preethi.reddi@emory.edu \\ Address: Department of Biology, 1510 Clifton Rd \#2006, Emory University, Atlanta, GA 30322, \\ USA \\ Jussi P. Posti, M.D., Ph.D. \\ Division of Clinical Neurosciences, Department of Neurosurgery, and Turku Brain Injury \\ Centre, Turku University Hospital, and University of Turku, Turku, Finland. \\ Email: jussi.posti@utu.fi \\ Address: Kiinamyllynkatu 4-8, FI-20521 Turku, Finland \\ Anna-Kerttu Kotilainen, B.M. \\ Faculty of Medicine and Life Sciences, University of Tampere, Tampere, Finland \\ Email: kotilainen.anna-kerttu.x@ student.uta.fi \\ Address: Faculty of Medicine and Life Sciences, University of Tampere, P.O. Box 100, FI- \\ 33014 University of Tampere, Tampere, Finland \\ Olli Tenovuo, M.D., Ph.D. \\ Turku Brain Injury Centre, Turku University Hospital, Finland \\ Address: Lemminkäisenkatu 3A; Teutori building, FI-20520 Turku, Finland \\ Juha Öhman, M.D., Ph.D. \\ Department of Neurosurgery, Tampere University Hospital and University of Tampere, \\ Tampere, Finland. \\ Email: juha.ohman@pshp.fi \\ Address: P.O. Box 2000, Teiskontie 35, FI-33521, Tampere, Finland \\ Henrik Zetterberg, M.D., Ph.D. \\ Institute of Neuroscience and Physiology, Department of Psychiatry and Neurochemistry, the \\ Sahlgrenska Academy at the University of Gothenburg, Mölndal, Sweden
}


Clinical Neurochemistry Laboratory, Sahlgrenska University Hospital, Mölndal, Sweden UK Dementia Research Institute at University College London, London, United Kingdom Department of Neurodegenerative Disease, University College London Institute of Neurology, Queen Square, London, United Kingdom Email: henrik.zetterberg@ clinchem.gu.se

Address: Blå Stråket 15, Vån 3 SU/Sahlgrenska, 41345 Göteborg, Sweden

Kaj Blennow, M.D., Ph.D.

Institute of Neuroscience and Physiology, Department of Psychiatry and Neurochemistry, the Sahlgrenska Academy at the University of Gothenburg, Mölndal, Sweden Clinical Neurochemistry Laboratory, Sahlgrenska University Hospital, Mölndal, Sweden Email: kaj.blennow@neuro.gu.se

Address: Blå Stråket 15, Vån 3 SU/Sahlgrenska, 41345 Göteborg, Sweden

Teemu M. Luoto, M.D., Ph.D.*

Department of Neurosurgery, Tampere University Hospital and University of Tampere, Tampere, Finland

Email: teemu.luoto@pshp.fi

Address: P.O. Box 2000, Teiskontie 35, FI-33521, Tampere, Finland

*Corresponding author:

Dr. Teemu M. Luoto

Tampere University Hospital, Department of Neurosurgery

P.O. Box 2000

Teiskontie 35

FI-33521 Tampere, Finland

Email: Teemu.Luoto@pshp.fi; Mobile: +358407039696 
Dear Editor,

Please find attached a manuscript entitled "Serum Neurofilament Light is Elevated Differentially in Older Adults with Uncomplicated Mild Traumatic Brain Injuries." There is tremendous interest in identifying serum biomarkers that have diagnostic or prognostic value following traumatic brain injury (TBI). However, some biomarkers used in TBI research, such as neurofilament light (NF-L), are elevated in people with neurological or neurodegenerative diseases. As such, it is critical to determine whether these biomarkers are useful for older adults who present with mild TBIs and who have pre-existing neurological conditions. This is the first study to do this. This study has not been previously published, and it has not been submitted to any other journal for peer review. We are hopeful that you will find this manuscript consistent with the objectives of Brain, and that you will initiate the peer review process.

Sincerely,

Grant L. Iverson, Ph.D.

Department of Physical Medicine and Rehabilitation, Harvard Medical School, and

Teemu Luoto, M.D., Ph.D.

Department of Neurosurgery, Tampere University Hospital

On behalf of our coauthors. 


\begin{abstract}
There is considerable interest in the use of blood biomarkers as diagnostic screening tests for mild traumatic brain injury (MTBI). Neurofilament light (NF-L) may have diagnostic and prognostic potential as a blood biomarker for MTBI. Elevated NF-L is associated with several neurological disorders associated with older age, however, which could confound its usefulness as a TBI biomarker. We examined whether NF-L is elevated differentially following uncomplicated MTBI in older adults with pre-injury neurological disorders. A sample of 118 adults (mean age $=62.3$ years, $\mathrm{SD}=22.5$, range $=18-100 ; 52.5 \%$ women) presenting to the emergency department (ED) of Tampere University Hospital with an uncomplicated MTBI were enrolled in this study. All had a Glasgow Coma Scale score of 14 or 15 . All participants underwent head CT in the ED and showed no macroscopic evidence of injury. Their most common mechanism of injury was a ground-level fall (70.3\%). Within 72 hours of injury, blood was collected from participants. The mean time between injury and blood sampling was 8.3 hours $(\mathrm{Md}=3.5 ; \mathrm{SD}=13.5 ; \mathrm{IQR}=1.9-6.0$, range=0.8-67.4, and 90\% collected within 19 hours $)$. A sample of 40 orthopedically-injured trauma control subjects recruited from a second ED also were examined. Serum NF-L levels were measured and analyzed using Human Neurology 4Plex A assay (N4PA) on a HD-1 Single molecule array (Simoa) instrument. A high correlation was found between age and NF-L levels in the total MTBI sample ( $\mathrm{r}=.80)$, within the subgroups without pre-injury neurological diseases $(r=.76)$ and with pre-injury neurological diseases $(\mathrm{r}=.68)$, and in the trauma control subjects $(\mathrm{r}=.76)$. Those with MTBIs and pre-injury neurological conditions had higher NF-L levels than those with no pre-injury neurological conditions $(\mathrm{p}<.001$, Cohen's $d=1.01)$. The subgroup with MTBIs aged $\geq 60$ had higher NF-L levels than those $<60(\mathrm{p}<.001, \mathrm{~d}=1.14)$. The subgroup of trauma controls aged 60 or older had
\end{abstract}


higher NF-L levels than those under the age of $60(\mathrm{p}<.001, \mathrm{~d}=1.67)$. For MTBI patients with no pre-injury neurological conditions, those aged $\geq 60$ had higher NF-L levels than those $<60$ $(\mathrm{p}<.001, \mathrm{~d}=1.18)$. For MTBI patients with pre-injury neurological conditions, those aged $\geq 60$ had higher NF-L levels than those $<60(\mathrm{p}<.001, \mathrm{~d}=1.12)$. Older age and pre-injury neurological diseases are associated with elevated serum NF-L levels during the first 72 hours following uncomplicated MTBI, which limits the value of this biomarker during this time period. Given the relatively slow temporal profile for the increase in NF-L, and sustained elevation, samples taken at later time points might be more clinically useful in older adults.

Key words: Head Injury; Traumatic Brain Injury; Biomarker; Blood; Neurofilament; Aging; Emergency Treatment

\section{Word Count: 409}




\section{Introduction}

Axonal damage is a pathological characteristic of traumatic brain injury (TBI), and it has been found to play a role in neuropsychological outcome (Warner et al., 2010). Neurofilaments are intermediate filaments (thread-like structures) (Ishikawa et al., 1968) that are involved in the growth and structure of axons (Eyer and Peterson, 1994). Neurofilament light (NF-L) is the smallest subunit of the neurofilament heteropolymer (a macromolecule composed of several bonded monomer subunits) (Lee et al., 1993). Together with the neurofilament medium and heavy subunits, NF-L is believed to facilitate scaffolding of the neural cytoskeleton-providing structural support for the axon and regulating axon diameter (Heins et al., 1993; Lee et al., 1993). NF-L plays a role in the structure of neurofilaments, assembly of the neurofilament heteropolymer, and formation of a stable stationary neurofilament network (Heins et al., 1993). NF-L is also involved in the transport of neurofilaments through the axon (Yates et al., 2009), axonal and dendritic branching and growth (Eyer and Peterson, 1994), and it is abundant in long subcortical white matter axons (Zetterberg et al., 2013). Proteolytic (i.e., enzyme breakdown) processes that occur during axonal degeneration lead to incomplete neurofilament degradation (Schlaepfer et al., 1984). Therefore, following axonal damage (Schlaepfer et al., 1984) or neuronal degeneration (Disanto et al., 2017), neurofilaments are released and are present in both the cerebrospinal fluid and the bloodstream (Bacioglu et al., 2016; Brureau et al., 2017).

There is steadily growing interest in examining NF-L following neurotrauma. NF-L levels have been found to increase in both cerebrospinal fluid (CSF) and blood during the first week(s) following TBI (Al Nimer et al., 2015; Shahim et al., 2016). They are elevated in those with traumatic structural abnormalities visible on computed tomography (CT) (Korley et al., 2018), highly elevated following severe TBI and diffuse axonal injury (Ljungqvist et al., 2017), 
and they are associated with outcome in patients with severe TBIs (Al Nimer et al., 2015; Shahim et al., 2016). NF-L is also elevated following mild neurotrauma, such as sport-related concussion (Shahim et al., 2018). Interestingly, in one study NF-L was reported to increase in American football players, over the course of a season, who had not experienced a symptomatic concussion (Oliver et al., 2016).

There has been a clarion call for more research focusing on TBI in older adults (Gardner et al., 2018). In Western countries, overall hospital admission rates for TBIs are decreasing, while admissions are increasing in patients over 65 years (Koskinen and Alaranta, 2008; Perez et al., 2012; Shivaji et al., 2014). Falls are a common cause of ED visits (Hoidrup et al., 2003; Johansen et al., 2011) and TBI (Helling et al., 1999; Sarani et al., 2009; Poyry et al., 2013) in older adults. Because older adults are more likely to have neurological conditions prior to brain injury, NF-L might be elevated differentially in older adults, compared to middle-aged or younger adults, following mild traumatic brain injury (MTBI). A number of studies indicate that serum NF-L levels are higher in people with diverse neurological and neurodegenerative diseases (Kuhle et al., 2016; Rohrer et al., 2016; Byrne et al., 2017; Disanto et al., 2017; Gattringer et al., 2017; Hansson et al., 2017; Kuhle et al., 2017; Mattsson et al., 2017; Novakova et al., 2017; Weston et al., 2017; De Marchis et al., 2018; Feneberg et al., 2018; Thompson et al., 2018; Wilke et al., 2018). Therefore, as a potential diagnostic or prognostic biomarker for neurotrauma, it is important to determine whether there is an association between age and NF-L levels in individuals who sustain MTBIs. The purpose of this study is to determine whether NF-L is elevated differentially in older adults with pre-existing neurological disorders or diseases who present to the emergency department (ED) following MTBI. We hypothesized that there would be an association between older age and NF-L levels in both orthopedically-injured trauma 
control subjects and patients who sustain uncomplicated MTBIs. In addition, we hypothesized that older adults who sustain MTBIs who have pre-existing neurological diseases would have the highest levels of NF-L.

\section{Participants}

\section{Methods}

A sample of 325 adults and older adults were evaluated in the ED of Tampere University Hospital (Tampere, Finland) following head trauma and enrolled in a study. Of those, 224 underwent head computed tomography (CT) and 190 had no trauma-related abnormalities identified on CT. Of the 190 with normal head CT scans, 130 had their blood sampled for longterm storage. For the final sample, 118 patients were included if their Glasgow Coma Scale score was 14 or 15 in the ED and they had their blood drawn within 72 hours of injury. The mean age of the sample was 62.3 years $(\mathrm{SD}=22.5$, Range $=18-100 ; 52.5 \%$ women $)$. Their mechanisms of injury were as follows: ground level fall $=70.3 \%$, fall from height or unclassified fall=7.6\%, violence $=5.9 \%$, sports $=5.1 \%$, bicycle accident $=3.4 \%$, motor vehicle accident $=2.5 \%$, motorcycle accident $=0.8 \%$, and other or unknown $=4.2 \%$. In the $E D$, a detailed case report form was completed for each enrolled patient. The percentages of the sample with loss of consciousness documented in the ED records were as follows: yes, witnessed $=17.8 \%$, suspected $=31.4 \%$, no $=41.5 \%$, and unknown $=9.3 \%$. The percentages of the sample with post-traumatic amnesia documented in the ED records were as follows: yes $=40.7 \%$, no $=53.4 \%$, and unknown $=5.9 \%$. The percentages with focal neurological signs in the ED were: yes $=10.2 \%$, no $=88.1 \%$, and unknown $=1.7 \%$. The health history of each enrolled patient was reviewed from the electronical patient records. Prior diagnosed diseases (including neurological diseases) were coded according to the ICD-10 classification (see Table 1). All patients provided written informed consent 
according to the Declaration of Helsinki. The study protocol was approved by the ethical review board of Pirkanmaa Hospital District, Finland (ethical code: R15045).

Table 1. Pre-injury neurological conditions identified in the centralized electronic medical records.

\begin{tabular}{|c|c|}
\hline & $\%(\mathrm{n})$ \\
\hline \multicolumn{2}{|l|}{ Mental and behavioral disorders (F01-99) } \\
\hline Organic, including symptomatic, mental disorders & $16.9(20)$ \\
\hline Vascular dementia (F01) & $0.8(1)$ \\
\hline Unspecified dementia (F03) & $5.9(7)$ \\
\hline \multicolumn{2}{|l|}{ Diseases of the nervous system (G00-99) } \\
\hline Cerebrovascular Disease & $16.1(19)$ \\
\hline Nontraumatic Subarachnoid Hemorrhage, status post & $1.7(2)$ \\
\hline Cerebral Infarction, status post & $15.3(18)$ \\
\hline Extrapyramidal and movement disorders (G20-26) & $3.4(4)$ \\
\hline Other degenerative diseases of the nervous system (G30-32) & $5.9(7)$ \\
\hline Alzheimer disease (G30) & $5.9(7)$ \\
\hline Episodic and paroxysmal disorders (G40-47) & $29.7(35)$ \\
\hline Epilepsy (G40) & $3.4(4)$ \\
\hline Transient cerebral ischemic attacks and related syndromes (G45) & $8.5(10)$ \\
\hline Nerve, nerve root and plexus disorders (G50-59) & $2.5(3)$ \\
\hline Polyneuropathies and other disorders of the peripheral nervous system (G60-64) & $1.7(2)$ \\
\hline Diseases of myoneural junction and muscle (G70-73) & $1.7(2)$ \\
\hline Cerebral palsy and other paralytic syndromes (G80-83) & $0.8(1)$ \\
\hline Other disorders of the nervous system (G90-99) & $0.8(1)$ \\
\hline Neoplasms (C00-D48) & \\
\hline Benign neoplasm of brain and other parts of central nervous system (D33) & $0.8(1)$ \\
\hline
\end{tabular}

Note: $\mathrm{N}=118$. Some subjects have more than one diagnosis. 
An orthopedically-injured control sample $(\mathrm{N}=40)$ was obtained from the ED of Turku University Hospital (Finland) and included a subgroup of a previously published sample (Posti et al., 2017). The orthopedic control sample included 22 men (55\%) and 18 women. Their mean age was 52.15 years old $(\mathrm{SD}=18.83, \mathrm{IQR}=35.75-64.75$, Range=22-90). The orthopedic injuries sustained by the control sample were as follows ( $n$, percentage of sample): simple ankle fracture $(11,27.5 \%)$, complex ankle fracture $(10,25.0 \%)$, wrist fracture $(5,12.5 \%)$, hip fracture $(3$, $7.5 \%)$, humerus fracture $(3,7.5)$, and forearm fracture $(2,5.0)$. One person in the sample $(2.5 \%)$ sustained each of the following injuries: clavicle fracture, complex pelvic fracture, knee fracture, hand fracture, and foot fracture. We were not able to determine whether some subjects experienced some degree of peripheral nerve injury. Control subjects were not included if there was any suspicion of an acute TBI (injury signs to the head, any suspicion of TBI signs at the time of injury, or symptoms suggesting a possible TBI) or they experienced polytrauma needing intensive care. After completion of the study, the health history of each enrolled patient was reviewed from the electronical patient records. One subject was identified as having cerebrovascular disease and one subject was identified as having Alzheimer's disease. Many of the control subjects underwent an MRI of their brain for research purposes (i.e., $n=31,77.5 \%$ of the sample). If on MRI they had clear evidence of small vessel ischemic disease, multiple white matter hyperintensities, small infarcts, or frontal cortical dysplasia (or possible low-grade glioma; $n=1)$ they were classified as have a pre-injury neurological disease $(n=14)$. Some incidental findings on MRI were classified as broadly normal, such as frontal calcification in the corpus callosum $(n=1)$ and venous angiomas or cavernoma $(n=3)$. The one subject with Alzheimer's disease documented in medical records did not undergo an MRI scan for research purposes. The control subjects were divided into three subgroups: no documented pre-injury 
neurological disease and broadly normal brain MRI scan $(n=16)$, no documented neurological disease and missing MRI scan (i.e., unknown pre-injury neurological disease; $n=9$ ), and those with pre-injury neurological disease $(n=15)$. The study protocol was approved by the ethical review board of the Hospital District of South-West Finland (code: 68/180/2011).

\section{Blood Sampling and Analytics}

Venous blood samples for the MTBI group were collected in the ED. The mean time between injury and blood sampling was 8.3 hours $(\mathrm{Md}=3.5 ; \mathrm{SD}=13.5 ; \mathrm{IQR}=1.9-6.0$, range=0.867.4, and $90 \%$ collected within 19 hours). For the trauma control sample, venous blood samples were collected on the day of injury or the following day. Serum NF-L levels were measured using the Human Neurology 4-Plex A assay (N4PA) on an HD-1 Single molecule array (Simoa) instrument according to instructions from the manufacturer (Quanterix, Lexington, MA). The measurements were performed by board-certified laboratory technicians who were blinded to clinical data. The limit of detection for NF-L was $0.104 \mathrm{pg} / \mathrm{mL}$ and the limit of quantification was $0.241 \mathrm{pg} / \mathrm{mL}$ with a calibration range of 0.533 to $453 \mathrm{pg} / \mathrm{mL}$. Two internal quality control (QC) samples were analyzed in each run. For a QC sample with a concentration of $13.9 \mathrm{pg} / \mathrm{mL}$, repeatability and intermediate precision was $4.4 \%$, while for a QC sample with a concentration of $7.1 \mathrm{pg} / \mathrm{mL}$, repeatability and intermediate precision was $6.1 \%$. For the trauma control sample with a concentration of $13.9 \mathrm{pg} / \mathrm{mL}$, repeatability and intermediate precision was _ $\%$, while for the sample with a concentration of $7.1 \mathrm{pg} / \mathrm{mL}$, repeatability and intermediate precision was

\%. The trauma control sample was run at a different time than the MTBI sample, and the two runs were not calibrated for each other.

\section{Statistical Analyses}


The associations between age and NF-L were examined in each group separately using Spearman correlations and nonparametric interferential statistics (Mann Whitney U tests) because both the biomarker levels and age were not normally distributed. Nonparametric analyses were also used to examine subgroups with the MTBI group with and without pre-injury neurological diseases. Receiver operator characteristic curve (ROC) analyses with a nonparametric estimate of the area under the curve were used to compare patients with MTBIs to trauma control subjects.

\section{Results}

Descriptive statistics for NF-L for the groups and subgroups are presented in Table 2. Within the orthopedically-injured trauma control group, the Spearman correlation between age and NF-L values was high ( $\mathrm{r}=.76)$. The subgroup aged 60 or older had higher NF-L levels than those under the age of $60(U=349, p<.001$, Cohen's $d=1.67$; also see Figure 1$)$. There was one extreme outlier in the trauma control group (NF-L level of $140 \mathrm{pg} / \mathrm{mL}$ ), a man in his mid-late 60s who was later found to have multiple periventricular white matter changes on brain MRI. The subgroup of control subjects with neurological disorders was older than the subgroup without neurological disorders $(\mathrm{U}=229, \mathrm{p}<.001, \mathrm{~d}=2.37)$, and they also had higher NF-L levels $(\mathrm{U}=184.5$, $\mathrm{p}=.011, \mathrm{~d}=1.59)$.

Within the uncomplicated MTBI group, there was no significant difference in NF-L levels between men and women $(\mathrm{U}=1,470, \mathrm{p}=.152)$. The Spearman correlation between age and NF-L values was high $(r=.80)$. The correlations between age and NF-L in subgroups of those with no pre-injury neurological conditions $(\mathrm{r}=.76)$ and pre-injury neurological conditions $(\mathrm{r}=.68)$ were high. Those with pre-injury neurological conditions had higher NF-L levels than those with no pre-injury neurological conditions ( $\mathrm{Md}=30.82$ vs. 12.64 , respectively; $\mathrm{U}=2,638, \mathrm{p}<.001$, 
$\mathrm{d}=1.01$ ). The subgroup aged 60 or older had higher NF-L levels than those under the age of 60 $(\mathrm{Md}=28.16$ vs. $7.52 ; \mathrm{U}=3,048, \mathrm{p}<.001, \mathrm{~d}=1.14)$. For those with no pre-injury neurological conditions, those age $\geq 60$ had higher NF-L levels than those age $<60$ (Md=19.80 vs. 7.15; $\mathrm{U}=850, \mathrm{p}<.001, \mathrm{~d}=1.18$ ). For those with pre-injury neurological conditions, those age $\geq 60$ had higher NF-L levels than those age $<60$ (Md=35.20 vs. 8.36; $\mathrm{U}=404, \mathrm{p}<.001, \mathrm{~d}=1.12)$.

A ROC analysis under a nonparametric assumption revealed a modest statistically significant area under the curve (AUC) estimate of $0.635(\mathrm{SE}=0.48, \mathrm{p}<.011,95 \% \mathrm{CI}=0.540-$ 0.730) for differentiating those with uncomplicated MTBIs from the trauma control subjects. However, when those with known pre-injury neurological diseases were excluded from the MTBI group only, there was no significant differentiation between the groups (AUC $=0.524$, $\mathrm{SE}=0.058, \mathrm{p}=.684,95 \% \mathrm{CI}=0.409-0.638)$. When those with known pre-injury neurological diseases were excluded from both groups, there was no significant differentiation between the groups (AUC=.601, $\mathrm{SE}=.065, \mathrm{p}=.139,95 \% \mathrm{CI}=0.474-0.729$ ), despite the fact that the MTBI group ( $\mathrm{n}=64)$ was older $(M=54.5$ years, $S D=23.1)$ than the control subjects $(\mathrm{n}=25 ; \mathrm{M}=43.7$ years, $\mathrm{SD}=16.3$ ). There was no significant differentiation between age-stratified subgroups with uncomplicated MTBIs and the trauma control subjects who were under the age of 60 $(\mathrm{AUC}=0.498, \mathrm{SE}=0.071, \mathrm{p}=981,95 \% \mathrm{CI}=0.360-0.637)$ or over the age of $60(\mathrm{AUC}=0.582$, $\mathrm{SE}=0.079, \mathrm{p}=.317,95 \% \mathrm{CI}=0.427-0.736)$. There was a significant differentiation between subgroups with uncomplicated MTBIs and the trauma control subjects, both of whom had preinjury neurological disease ( $\mathrm{AUC}=.678, \mathrm{SE}=.076, \mathrm{p}=.036,95 \% \mathrm{CI}=0.528-0.827)$. 
Table 2. Descriptive statistics for NF-L for the groups and subgroups.

\begin{tabular}{|c|c|c|c|c|c|c|}
\hline Sample & $\mathrm{N}$ & M & Md & SD & IQR & Range \\
\hline Total Orthopedically-Injured Control Sample & 40 & 18.30 & 10.65 & 23.35 & $6.83-20.68$ & $4.10-141.30$ \\
\hline Women & 18 & 11.81 & 9.55 & 10.53 & $6.48-12.03$ & $4.10-51.20$ \\
\hline Men & 22 & 23.60 & 13.15 & 29.28 & $7.90-26.90$ & $4.60-141.30$ \\
\hline Age $<60$ & 25 & 8.94 & 8.20 & 4.20 & $5.80-11.20$ & $4.10-23.50$ \\
\hline Age $\geq 60$ & 15 & 33.89 & 24.40 & 32.74 & $16.70-44.70$ & $9.80-141.30$ \\
\hline No Pre-Injury Neurological Conditions* & 16 & 9.41 & 7.55 & 4.99 & $5.65-13.05$ & $4.10-23.50$ \\
\hline Unknown Pre-Injury Neurological Conditions* & 9 & 19.43 & 10.30 & 17.20 & $8.95-35.10$ & $5.40-51.20$ \\
\hline Pre-Injury Neurological Conditions* & 15 & 27.09 & 17.60 & 33.91 & $9.80-31.10$ & $4.60-141.30$ \\
\hline Total Uncomplicated MTBI Sample & 118 & 32.07 & 18.97 & 40.43 & $9.05-35.40$ & $2.62-246.92$ \\
\hline Men & 56 & 28.79 & 15.05 & 40.33 & $7.52-31.11$ & $2.62-237.30$ \\
\hline Women & 62 & 35.04 & 20.88 & 40.62 & $10.29-47.06$ & $3.40-246.92$ \\
\hline Age $<60$ & 46 & 10.33 & 7.52 & 7.80 & $5.54-11.49$ & $2.62-36.44$ \\
\hline Age $\geq 60$ & 72 & 45.96 & 28.16 & 46.40 & $18.42-51.01$ & $9.06-246.92$ \\
\hline No Pre-Injury Neurological Conditions & 64 & 17.16 & 12.64 & 14.93 & $7.04-21.60$ & $2.62-85.44$ \\
\hline Pre-Injury Neurological Conditions & 54 & 49.75 & 30.82 & 52.50 & $16.96-68.47$ & $3.40-246.92$ \\
\hline Age $<60$ No Pre-Injury Neurological Conditions & 37 & 11.00 & 7.15 & 8.49 & $5.51-13.08$ & $2.62-36.44$ \\
\hline Age $<60$ Pre-Injury Neurological Conditions & 9 & 7.58 & 8.36 & 2.73 & $4.89-9.56$ & $3.40-11.69$ \\
\hline Age $\geq 60$ No Pre-Injury Neurological Conditions & 27 & 25.60 & 19.80 & 17.67 & $14.84-31.92$ & $9.06-85.44$ \\
\hline Age $\geq 60$ Pre-Injury Neurological Conditions & 45 & 58.18 & 35.20 & 53.69 & $23.18-86.21$ & $10.11-246.92$ \\
\hline
\end{tabular}

Note: *The average ages for control subjects were as follows: No Neurological Conditions $=\mathrm{M}=36.2, \mathrm{Md}=33.5, \mathrm{SD}=11.5$; Neurological Conditions Unknown=M=57.1, $\mathrm{Md}=55.0, \mathrm{SD}=15.4$; and Pre-Injury Neurological Conditions=M=66.2, $\mathrm{Md}=65.0, \mathrm{SD}=13.9$.

Figure 1 illustrates every individual subjects' serum NF-L level. The subjects are sorted by age, beginning with age 18 and ending with age 100. Notice that serum NF-L levels are well below 20 for the large majority of people in all three groups under the age of 60 . As seen in 
Table 2, 75\% of those with MTBIs who are under the age of 60 have NF-L values less than 11.5 (see the IQR). In contrast, individuals in their 70s, 80s, and 90s have highly elevated NF-L levels compared to young and middle-aged adults. Older adults with pre-injury neurological conditions, in general, have higher NF-L levels than older adults who do not have pre-injury neurological conditions (see Table 2 and Figure 1). 
Figure 1. Neurofilament light levels in adults and older adults sorted by age. (Original graph)

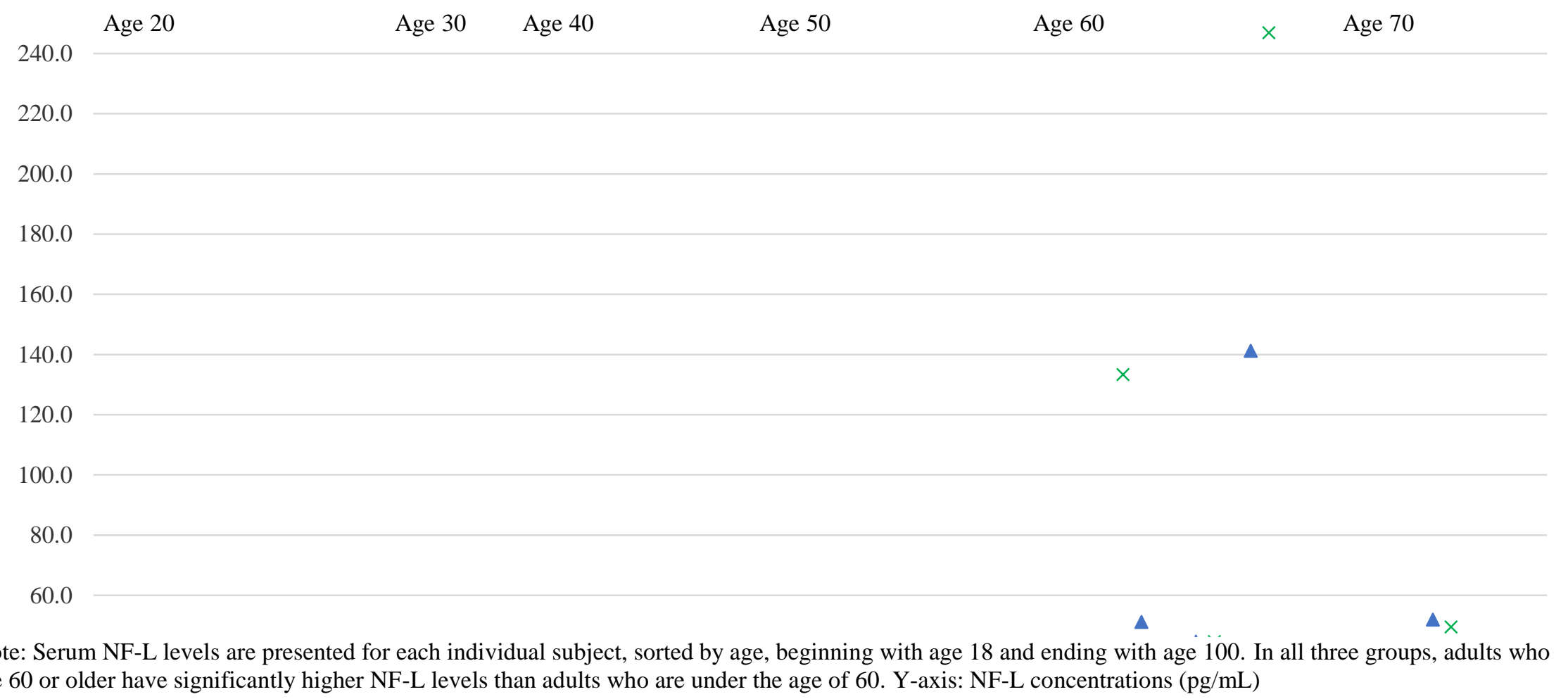
are 60 or older have significantly higher NF-L levels than adults who are under the age of 60 . Y-axis: NF-L concentrations (pg/mL) 
Figure 1. Neurofilament light levels in adults and older adults sorted by age. (New Graph)

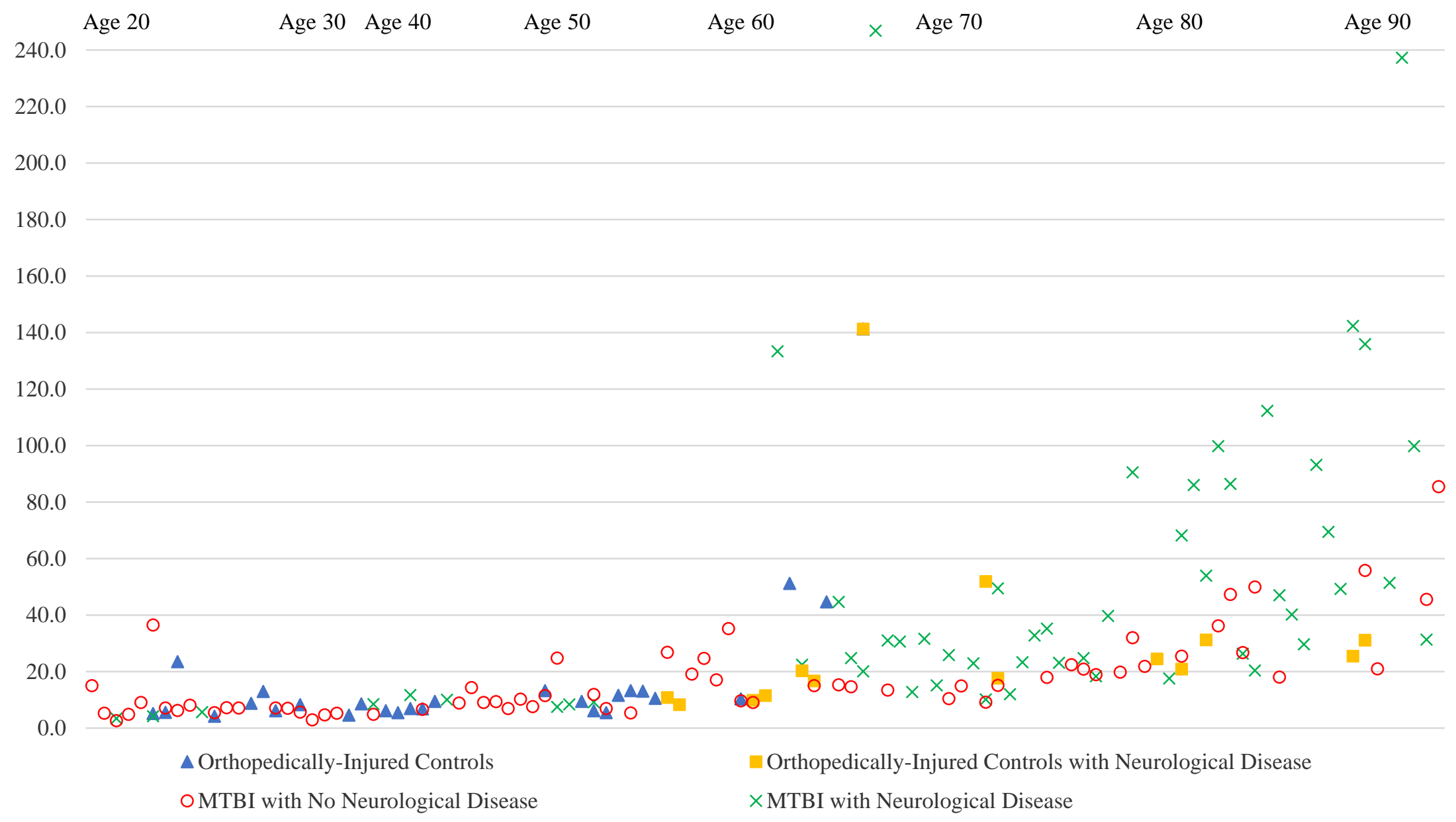

Note: Serum NF-L levels are presented for each individual subject, sorted by age, beginning with age 18 and ending with age 100. Y-axis: NF-L concentrations $(\mathrm{pg} / \mathrm{mL})$. The patients with MTBIs who had neurological diseases had those diseases documented in their medical records. In contrast, the orthopedically-injured control subjects were classified as having neurological diseases if they had an MRI for research purposes and that MRI showed evidence of white matter ischemic disease and/or microinfarcts. 


\section{Discussion}

There is considerable interest in understanding the diagnostic and prognostic potential of NF-L as a serum biomarker for axonal injury associated with TBI (Al Nimer et al., 2015; Shahim et al., 2016; Korley et al., 2018). It is recognized that TBI in older adults is understudied (Gardner et al., 2018), and the effects of neurotrauma on the aging brain are not well understood. It is essential to examine NF-L in association with aging and neurological diseases because these factors might fundamentally compromise its usefulness as biomarker for TBI in older adults. Our study is the first to examine whether NF-L is elevated differentially following uncomplicated MTBI in older adults with pre-existing neurological disorders. As hypothesized, we found that age is associated with NF-L levels in subgroups of individuals with MTBIs and no pre-injury neurological disorders and in those with pre-injury neurological disorders. Specifically, individuals age $\geq 60$ with MTBIs had higher NF-L levels than individuals under the age of 60 for those with pre-injury neurological disorders and for those without pre-injury neurological disorders. We also found that, overall, individuals with MTBIs and pre-injury neurological disorders had higher NF-L levels than individuals without pre-injury neurological disorders. The strong association between older age and higher NF-L levels was also present in the orthopedically-injured trauma control sample. In general, NF-L was not particularly useful for differentiating those with uncomplicated MTBIs from trauma control subjects.

Our results are consistent with past studies showing that serum levels of NF-L are higher in people with diverse neurological and neurodegenerative conditions. Researchers have reported that serum levels of NF-L are higher in people with multiple sclerosis (Kuhle et al., 2016; Disanto et al., 2017; Kuhle et al., 2017; Novakova et al., 2017), acute ischemic stroke (De Marchis et al., 2018), active cerebral small vessel disease (Gattringer et al., 2017), familial 
Alzheimer's disease (Mattsson et al., 2017; Weston et al., 2017), Huntington's disease (Byrne et al., 2017), frontotemporal dementia (Rohrer et al., 2016), Creutzfeldt-Jakob disease (Thompson et al., 2018), Parkinsonian disorders (Hansson et al., 2017), other degenerative ataxias such as multiple system atrophy (Wilke et al., 2018) and amyotrophic lateral sclerosis (Feneberg et al., 2018).

Regarding clinical usefulness, there are no established and validated cutoff values for NF-L as a biomarker for TBI, of any severity, across the lifespan, using any commerciallyavailable assay. It is important to note that studies suggest that NF-L has quite slow serum level dynamics. After acute injury it appears to reach its peak concentration more than 7 days after the injury (Shahim et al., 2016; Shahim et al., 2018). NF-L may therefore be of limited use in the Emergency Department but may prove to be a useful biomarker for predicting clinical outcome with samples taken several days following injury (e.g., day 7-10). Future researchers can address the methodological limitations of the present study, and gaps in the literature more broadly, by (i) examining NF-L levels in older adults stratified by brain injury severity (i.e., mild, moderate, and severe); (ii) studying the temporal kinetics of NF-L in the bloodstream after TBI to determine reliable time windows for acute diagnostics of TBI and outcome prediction (across the lifespan); (iii) determining if NF-L levels can differentiate older adults with mild head trauma and positive or negative day-of-injury head computed tomography findings; and (iv) examining if extracerebral and especially peripheral nerve injuries affect the clinical reliability of NF-L in TBI diagnostics. Much additional research is needed to determine whether NF-L is useful for diagnostic or prognostic purposes following TBIs of all severities in older adults. 
Funding: The study was financially supported by the Finnish State Research Funding, and the Finnish Medical Society Duodecim. Dr. Luoto and Dr. Posti have received funding from Government's Special Financial Transfer tied to academic research in Health Sciences (Finland). Dr. Posti has received funding from the Emil Aaltonen Foundation sr and the Finnish Brain Foundation sr. Dr. Blennow acknowledges funding from The Torsten Söderberg Foundation, the Swedish Research Council, and the Swedish Brain Foundation. Dr. Zetterberg is a Wallenberg Academy Fellow and acknowledges support from the Swedish and European Research Council and the Dementia Research Institute at UCL.

Acknowledgements: The authors acknowledge research assistant Anne Simi for her assistance with the patient enrolment and data collection at Tampere University Hospital, and research coordinator Annamari Aitolahti for her assistance with blood sample logistics. The authors thank TBIcare investigators Riikka S.K. Takala, Ari J. Katila, Janek Frantzén, Henna Ala-Seppälä, Anna Kyllönen, Henna-Riikka Maanpää and Jussi Tallus for their contribution in control patient recruitment at Turku University Hospital.

Author Disclosure Statement: Grant Iverson acknowledges unrestricted philanthropic support from the Mooney-Reed Charitable Foundation and ImPACT Applications, Inc. He serves as a strategic scientific advisor for BioDirection, Inc. Jussi Posti has received speaker's fees from Orion corporation and Finnish Medical Association. Dr. Blennow has served as a consultant or at advisory boards for Alzheon, BioArctic, Biogen, Eli Lilly, Fujirebio Europe, IBL International, Merck, Novartis, Pfizer, and Roche Diagnostics, and is a co-founder of Brain Biomarker Solutions in Gothenburg AB, a GU Venture-based platform company at the University of Gothenburg. 


\section{Considerations}

We mention that these groups were analyzed in separate runs and that the results were not calibrated. However, there is no discussion whether this could influence our results.

Further still, I am not sure if the age should be included in the analysis in other ways than as a correlation. Dividing the patients to those above and below 60 is arbitrary and has no biological basis. Correlation between age and NF-L levels is more informative, together with figure 1. There are many assays in routine clinical use where the normative values are age-adjusted. What comes to TBI biomarkers, NF-L is not an exception, similar problems have been found e.g. with S100B (Fluids Barriers CNS. 2016 Nov 30;13(1):21). I guess we should simply state that if NF$\mathrm{L}$ will be developed for clinical use in TBI, the normative values should probably be ageadjusted and that the results may not be reliable in those with pre-existing neurological diseases.

GI says: visual inspection of the age-ordered data in Figure 1, and the strong correlation between age and NF-L, is very interesting. I agree that it is somewhat arbitrary to choose 60 to create groups. 


\section{References}

Al Nimer F, Thelin E, Nystrom H, Dring AM, Svenningsson A, Piehl F, et al. Comparative Assessment of the Prognostic Value of Biomarkers in Traumatic Brain Injury Reveals an Independent Role for Serum Levels of Neurofilament Light. PLoS ONE 2015; 10: e0132177.

Bacioglu M, Maia LF, Preische O, Schelle J, Apel A, Kaeser SA, et al. Neurofilament Light Chain in Blood and CSF as Marker of Disease Progression in Mouse Models and in Neurodegenerative Diseases. Neuron 2016; 91: 56-66.

Brureau A, Blanchard-Bregeon V, Pech C, Hamon S, Chaillou P, Guillemot JC, et al. NF-L in cerebrospinal fluid and serum is a biomarker of neuronal damage in an inducible mouse model of neurodegeneration. Neurobiol Dis 2017; 104: 73-84.

Byrne LM, Rodrigues FB, Blennow K, Durr A, Leavitt BR, Roos RAC, et al. Neurofilament light protein in blood as a potential biomarker of neurodegeneration in Huntington's disease: a retrospective cohort analysis. Lancet Neurol 2017; 16: 601-609.

De Marchis GM, Katan M, Barro C, Fladt J, Traenka C, Seiffge DJ, et al. Serum neurofilament light chain in patients with acute cerebrovascular events. Eur J Neurol 2018; 25: 562-568.

Disanto G, Barro C, Benkert P, Naegelin Y, Schadelin S, Giardiello A, et al. Serum Neurofilament light: A biomarker of neuronal damage in multiple sclerosis. Ann Neurol 2017; 81: 857-870.

Eyer J, Peterson A. Neurofilament-deficient axons and perikaryal aggregates in viable transgenic mice expressing a neurofilament-beta-galactosidase fusion protein. Neuron 1994; 12: $389-405$. 
Feneberg E, Oeckl P, Steinacker P, Verde F, Barro C, Van Damme P, et al. Multicenter evaluation of neurofilaments in early symptom onset amyotrophic lateral sclerosis. Neurology 2018; 90: e22-e30.

Gardner RC, Dams-O'Connor K, Morrissey MR, Manley GT. Geriatric Traumatic Brain Injury: Epidemiology, Outcomes, Knowledge Gaps, and Future Directions. J Neurotrauma 2018.

Gattringer T, Pinter D, Enzinger C, Seifert-Held T, Kneihsl M, Fandler S, et al. Serum neurofilament light is sensitive to active cerebral small vessel disease. Neurology 2017; 89: 2108-2114.

Hansson O, Janelidze S, Hall S, Magdalinou N, Lees AJ, Andreasson U, et al. Blood-based NfL: A biomarker for differential diagnosis of parkinsonian disorder. Neurology 2017; 88: 930-937.

Heins S, Wong PC, Muller S, Goldie K, Cleveland DW, Aebi U. The rod domain of NF-L determines neurofilament architecture, whereas the end domains specify filament assembly and network formation. J Cell Biol 1993; 123: 1517-33.

Helling TS, Watkins M, Evans LL, Nelson PW, Shook JW, Van Way CW. Low falls: an underappreciated mechanism of injury. J Trauma 1999; 46: 453-6.

Hoidrup S, Sorensen TI, Gronbaek M, Schroll M. Incidence and characteristics of falls leading to hospital treatment: a one-year population surveillance study of the Danish population aged 45 years and over. Scand J Public Health 2003; 31: 24-30.

Ishikawa H, Bischoff R, Holtzer H. Mitosis and intermediate-sized filaments in developing skeletal muscle. J Cell Biol 1968; 38: 538-55. 
Johansen A, Dickens J, Jones M, Richmond P, Evans R. Emergency department presentation following falls: development of a routine falls surveillance system. Emerg Med J 2011; 28: $25-8$.

Korley FK, Yue JK, Wilson D, Hrusovsky K, Diaz-Arrasta R, Ferguson AR, et al. Performance Evaluation of a Multiplex Assay for Simultaneous Detection of Four Clinically Relevant TBI Biomarkers. J Neurotrauma 2018.

Koskinen S, Alaranta H. Traumatic brain injury in Finland 1991-2005: a nationwide register study of hospitalized and fatal TBI. Brain Inj 2008; 22: 205-14.

Kuhle J, Barro C, Disanto G, Mathias A, Soneson C, Bonnier G, et al. Serum neurofilament light chain in early relapsing remitting MS is increased and correlates with CSF levels and with MRI measures of disease severity. Mult Scler 2016; 22: 1550-1559.

Kuhle J, Nourbakhsh B, Grant D, Morant S, Barro C, Yaldizli O, et al. Serum neurofilament is associated with progression of brain atrophy and disability in early MS. Neurology 2017; 88: 826-831.

Lee MK, Xu Z, Wong PC, Cleveland DW. Neurofilaments are obligate heteropolymers in vivo. J Cell Biol 1993; 122: 1337-50.

Ljungqvist J, Zetterberg H, Mitsis M, Blennow K, Skoglund T. Serum Neurofilament Light Protein as a Marker for Diffuse Axonal Injury: Results from a Case Series Study. J Neurotrauma 2017; 34: 1124-1127.

Mattsson N, Andreasson U, Zetterberg H, Blennow K, Alzheimer's Disease Neuroimaging I. Association of Plasma Neurofilament Light With Neurodegeneration in Patients With Alzheimer Disease. JAMA neurology 2017; 74: 557-566. 
Novakova L, Zetterberg H, Sundstrom P, Axelsson M, Khademi M, Gunnarsson M, et al.

Monitoring disease activity in multiple sclerosis using serum neurofilament light protein. Neurology 2017; 89: 2230-2237.

Oliver JM, Jones MT, Kirk KM, Gable DA, Repshas JT, Johnson TA, et al. Serum Neurofilament Light in American Football Athletes over the Course of a Season. J Neurotrauma 2016; 33: 1784-1789.

Perez K, Novoa AM, Santamarina-Rubio E, Narvaez Y, Arrufat V, Borrell C, et al. Incidence trends of traumatic spinal cord injury and traumatic brain injury in Spain, 2000-2009. Accid Anal Prev 2012; 46: 37-44.

Posti JP, Hossain I, Takala RS, Liedes H, Newcombe V, Outtrim J, et al. Glial Fibrillary Acidic Protein and Ubiquitin C-Terminal Hydrolase-L1 Are Not Specific Biomarkers for Mild CT-Negative Traumatic Brain Injury. J Neurotrauma 2017.

Poyry T, Luoto TM, Kataja A, Brander A, Tenovuo O, Iverson GL, et al. Acute assessment of brain injuries in ground-level falls. J Head Trauma Rehabil 2013; 28: 89-97.

Rohrer JD, Woollacott IO, Dick KM, Brotherhood E, Gordon E, Fellows A, et al. Serum neurofilament light chain protein is a measure of disease intensity in frontotemporal dementia. Neurology 2016; 87: 1329-36.

Sarani B, Temple-Lykens B, Kim P, Sonnad S, Bergey M, Pascual JL, et al. Factors associated with mortality and brain injury after falls from the standing position. J Trauma 2009; 67: 954-8.

Schlaepfer WW, Lee C, Trojanowski JQ, Lee VM. Persistence of immunoreactive neurofilament protein breakdown products in transected rat sciatic nerve. J Neurochem 1984; 43: 85764. 
Shahim P, Gren M, Liman V, Andreasson U, Norgren N, Tegner Y, et al. Serum neurofilament light protein predicts clinical outcome in traumatic brain injury. Scientific reports 2016; 6: 36791 .

Shahim P, Tegner Y, Marklund N, Blennow K, Zetterberg H. Neurofilament light and tau as blood biomarkers for sports-related concussion. Neurology 2018; 90: e1780-e1788.

Shivaji T, Lee A, Dougall N, McMillan T, Stark C. The epidemiology of hospital treated traumatic brain injury in Scotland. BMC Neurol 2014; 14: 2.

Thompson AGB, Luk C, Heslegrave AJ, Zetterberg H, Mead SH, Collinge J, et al.

Neurofilament light chain and tau concentrations are markedly increased in the serum of patients with sporadic Creutzfeldt-Jakob disease, and tau correlates with rate of disease progression. J Neurol Neurosurg Psychiatry 2018.

Warner MA, Marquez de la Plata C, Spence J, Wang JY, Harper C, Moore C, et al. Assessing spatial relationships between axonal integrity, regional brain volumes, and neuropsychological outcomes after traumatic axonal injury. J Neurotrauma 2010; 27: 2121-30.

Weston PSJ, Poole T, Ryan NS, Nair A, Liang Y, Macpherson K, et al. Serum neurofilament light in familial Alzheimer disease: A marker of early neurodegeneration. Neurology 2017; 89: 2167-2175.

Wilke C, Bender F, Hayer SN, Brockmann K, Schols L, Kuhle J, et al. Serum neurofilament light is increased in multiple system atrophy of cerebellar type and in repeat-expansion spinocerebellar ataxias: a pilot study. J Neurol 2018. 
Yates DM, Manser C, De Vos KJ, Shaw CE, McLoughlin DM, Miller CC. Neurofilament subunit (NFL) head domain phosphorylation regulates axonal transport of neurofilaments. Eur J Cell Biol 2009; 88: 193-202.

Zetterberg H, Smith DH, Blennow K. Biomarkers of mild traumatic brain injury in cerebrospinal fluid and blood. Nat Rev Neurol 2013; 9: 201-10. 